\title{
Air quality modelling as a tool for sustainable urban traffic management
}

\author{
J. H. Amorim, M. Lopes, C. Borrego, R. Tavares \& A. I. Miranda \\ CESAM, Department of Environment and Planning, \\ University of Aveiro, Portugal
}

\begin{abstract}
This work intends to assess the impact of road traffic management on urban air quality at the street level. At the core of the applied methodology is the numerical modelling of wind flow and air pollutants dispersion in a typical European urban environment. Through the application of a Computational Fluid Dynamics (CFD) model, the synergies between the 3D configuration of the street-canyon, in addition to the impacts on the dispersion of CO and PM10 emitted by vehicles, were evaluated. Air quality simulations were performed for a study domain of approximately $250 \times 1000 \mathrm{~m}^{2}$ in the downtown of a medium sized Portuguese town, with average daily traffic of 21,400 vehicles. Different traffic scenarios were analysed based on the modification of the number and location of traffic lanes for typical meteorological boundary conditions. Complex dispersion patterns were obtained due to the effect of buildings and trees on the wind flow. Moreover, simulations for the different scenarios have shown distinct behaviours of the air pollutants due to the prevailing wind direction and road positioning. It was concluded that, for typical conditions, the closure of the two traffic lanes located on the south side of the main avenue and the two adjacent streets is the best solution for air quality improvement. This scenario leads to a $31 \%$ reduction in CO and PM10 concentrations on the North sidewalk and 81\% on the South sidewalk when comparing to present conditions. The other alternative leads to a reduction of, respectively, $70 \%$ and $20 \%$. The first scenario also leads to maximum decreases of $57 \%$ and $59 \%$, respectively, in the $\mathrm{CO}$ and PM10 peak concentrations. These conclusions stress the importance of integrating the knowledge provided by the application of CFD models in urban planning and road traffic management with the goal of promoting urban sustainability.
\end{abstract}


Keywords: road traffic management, traffic scenarios, traffic emissions, CFD modelling, street canyon, air pollution, human comfort, human health, hot-spots.

\section{Introduction}

Currently, $80 \%$ of European citizens live in cities and towns, many of them characterised by intense road traffic and air pollution [1]. The official statistics and reports show that the trends on urban sprawl and road transportation have significant effects on human health. Nearly 100.000 deaths occur, each year, in the largest European cities as a consequence of air pollution [2]. In developed countries, $20 \%$ of respiratory infections are attributed to environmental causes (indoor and outdoor pollution), rising to $42 \%$ in developing countries [3].

In recent decades the study of urban structure impacts on the quality of life and on the environment has become a key issue for urban sustainability. Several studies recognise the importance of urban planning for the improvement of the interactions between different land uses and economic activities, and also towards a more sustainable urban metabolism [4]. Urban structure (sprawl or compact) is intimately related with urban fluxes (incoming and outgoing) of material, energy, information, people, etc. A major interest lies in understanding the role of planning on induced mobility patterns and thereafter on air quality, particularly related to the increasing use of private cars. As referred to by Oke [5] on seminal work on street design and the urban canopy layer climate, planning is always involved in making choices between alternatives.

In this context, the current challenge to urban planners and environmental engineers is to reverse the impacts on environment and health resulting from the problematic cohabitation between intense road traffic and high population densities, as a way to promote a better quality of life to urban populations. The rapid and continuous growth of hardware capabilities opens a vast number of new possibilities for CFD models, even in applications within urban environments, where the simulation of three-dimensional (3D) flow and dispersion fields represents an extremely demanding task due to the highly complex geometries present.

The main purpose of this work is to assess the impact of different road traffic management strategies on urban air quality through the application of a CFD model to a specific case study - a medium sized town in central Portugal.

\section{CFD model}

The CFD model used for this study is the multi-purpose commercial software FLUENT®, version 6.1.18 for UNIX platforms. This model has been applied in the simulation of flow and dispersion of fluids and particles within confined and open complex geometries in different scientific and technical domains. In particular, it has been previously validated by the authors for urban air quality modelling purposes [6-8]. 
In the spatial discretisation of the computational domain an unstructured meshing scheme was used. For the simulation of 3D flow and dispersion an Eulerian approach was applied, assuming steady-state conditions, while for the turbulence closure, a k- $\varepsilon$ model was used. Two road traffic-emitted pollutants were considered: carbon monoxide (CO) and particles of 10 micrometers or less (PM10). As a simplification, no chemical reactions were considered and the air pollutants removal by the canopy was neglected.

In terms of boundary conditions a no-slip condition was imposed at all solid surfaces. The flow in the near-wall region is represented through the law-of-thewall for mean velocity (for more details see [9]). At the top a symmetry boundary was defined, assuming a zero flux of all quantities across this horizontal plane. At the inlets (defined according to the wind direction), the wind velocity variation with height was calculated through the application of the power-law vertical wind profile (using a power-law exponent equal to 0.4 , which is typical for well-developed urban areas under near-neutral conditions).

Road traffic emissions of CO and PM10 were estimated applying the Transport Emission Model for Line Sources (TREM) developed by Borrego et al. [6], which is based on the MEET/COST methodology and uses detailed data on traffic flow from vehicles counting.

\section{Case study}

The case study selected is located at the centre of a medium sized town in Central Portugal, Aveiro.

\subsection{General description}

Located at the Norwest Portuguese coastline at $78 \mathrm{~km}$ from Porto and $275 \mathrm{~km}$ from Lisbon city, Aveiro is an important industrial and commercial area and a strategic point for international trade, benefiting from several transport networks, including road, rail and maritime connections. The municipality of Aveiro covers an area of $200 \mathrm{~km}^{2}$, it has a population of 75,000 inhabitants, and a university campus with nearly 15,000 students.

\subsection{Climate}

The climate of the Aveiro region is strongly influenced by the proximity of the ocean (at around $6 \mathrm{~km}$ ) and the events related to atmospheric dynamics, such as weather fronts and sea breezes, and geographical factors. It is characterized by a temperate Mediterranean climate, with hot and dry summers, and cold and rainy winters. The annual temperature is around $15.5^{\circ} \mathrm{C}$, while the average annual cumulative precipitation level is $908 \mathrm{~mm}$. From data recorded between 1981 and 1995 at the University of Aveiro meteorological tower (coordinates: $40^{\circ} 27^{\prime} 38^{\prime \prime} \mathrm{N}$, $8^{\circ} 43^{\prime} 18^{\prime \prime} \mathrm{W}$; elevation: $3.5 \mathrm{~m}$ ), it can be inferred that the average wind speed is minimal in summer, at 9:00, and maximal in spring, at 15:00. The mean wind speed in the morning is $9.5 \mathrm{~km} \cdot \mathrm{h}^{-1}$, rising up to $16.5 \mathrm{~km} \cdot \mathrm{h}^{-1}$ in the afternoon. The 
(a)

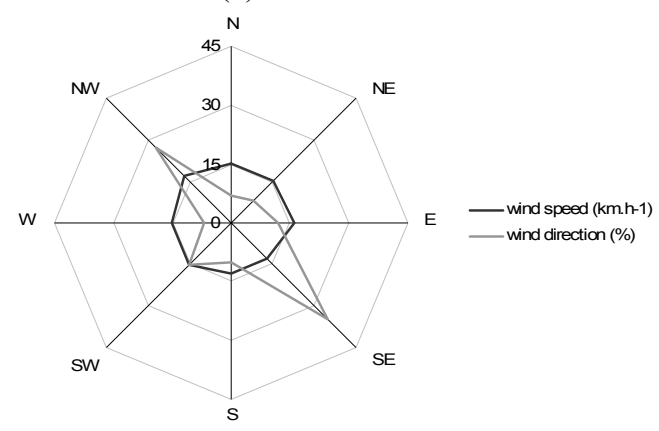

(b)

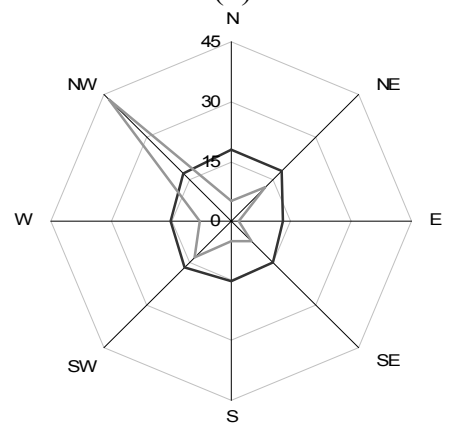

Figure 1: Wind roses for Aveiro city at (a) 9:00 and (b) 15:00, for the period between 1981 and 1995 .

(a)

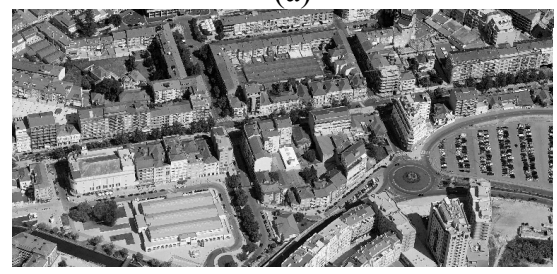

(b)

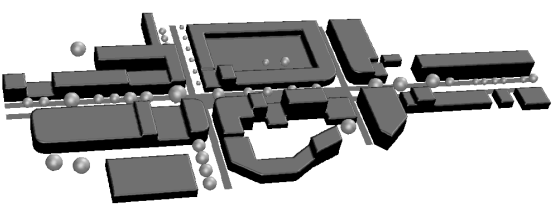

Figure 2: (a) Satellite image of the study domain, and (b) computational domain generated for the set of buildings and trees in the study area.

most common wind direction throughout the year is, in the morning, the Northwest (NW) and Southeast (SE), and in the afternoon is the NW, as can be seen in the wind roses shown in Figure 1.

\subsection{Computational domain}

The computational domain, with approximately $250 \times 1000 \mathrm{~m}^{2}$, was defined at the centre of Aveiro, in an area with residential and commercial characteristics (see Figure 2). It is centred at one of the most important thoroughfares of the town, the "Lourenço Peixinho" (LP) Avenue, which makes the connection between the western coastland areas and the national road network in the eastern side of the city, and is a privileged area for services and commerce. This area is characterised by the existence of a significant number of dense tall trees, in particular along the entire extension of the central axis of the Avenue. Figure 2 also shows the 3D geometry created using an unstructured meshing scheme from FLUENT's pre-processor Gambit, resulting in a total of nearly 2 million computational cells. 


\subsection{Road traffic flux and emissions}

The main emission source in the computational domain is the LP Avenue, which presently has an average daily traffic of 21,400 vehicles distributed by four lanes, two in each sense, separated by a row of dense and tall trees. Four other streets were also considered.

For the estimation of typical CO and PM10 emissions within this area, a traffic counting campaign was carried out between the $3^{\text {rd }}$ and the $24^{\text {th }}$ of October 2009. A total of eight monitoring sites were located in the previously mentioned roads. In the case of the LP Avenue the two senses were monitored. Vehicles were aggregated according to the following categories: passenger cars, light duty vehicles, heavy duty vehicles and urban busses. Additionally to these different categories a set of classes for a more detailed vehicle classification was implemented in the TREM model allowing to distinguish fuel type (gasoline, diesel, LPG), engine capacity, and technology (emission standards implementation linked to vehicle age). Mean hourly values of the number of vehicles were calculated allowing one to define typical traffic fluxes for weekdays and weekends in this thoroughfare. CO and PM10 emissions were then calculated with TREM. Results are shown in Figure 3.

(a)

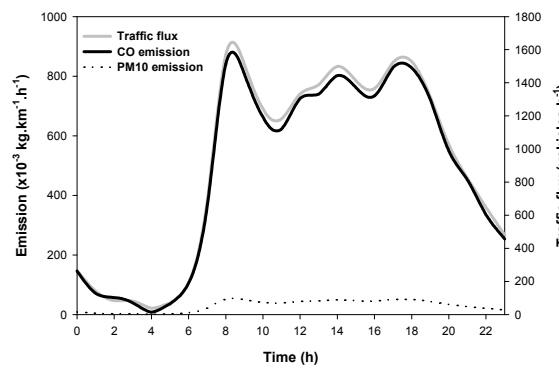

(b)

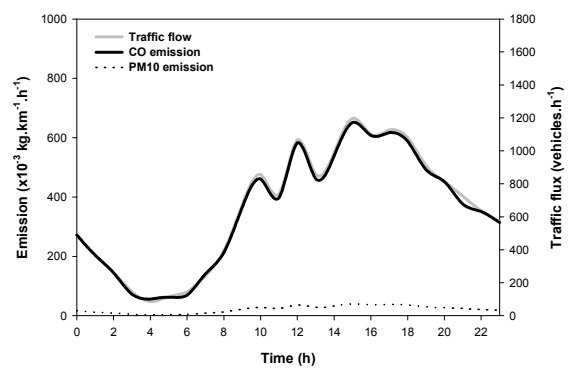

Figure 3: Time evolution of mean hourly values of the total number of vehicles, CO emission $\left(\times 10^{-3} \mathrm{~kg} \cdot \mathrm{km}^{-1} \cdot \mathrm{h}^{-1}\right)$ and PM10 emission $\left(\times 10^{-3} \mathrm{~kg} \cdot \mathrm{km}^{-1} \cdot \mathrm{h}^{-1}\right)$ for a typical (a) week day and (b) weekend day.

Differences are found for traffic flows along week and weekend days. In a typical week day, three distinct peaks occur: in the early morning, midday, and in the late afternoon; while in the weekend there is not a clear peak, as expected. On the other hand, during the night period the traffic flux is higher in the weekends. In general, the peak mean hourly traffic values in a week day are approximately $50 \%$ higher than the ones monitored in the weekend.

In terms of TREM outputs, in a typical week day, traffic emissions vary between a minimum of $3 \times 10^{-3} \mathrm{~kg} \cdot \mathrm{km}^{-1} \cdot \mathrm{h}^{-1}$ for $\mathrm{CO}$ and $1 \times 10^{-3} \mathrm{~kg} \cdot \mathrm{km}^{-1} \cdot \mathrm{h}^{-1}$ for PM10 at 4:00, corresponding to a total of 14 vehicles. $\mathrm{h}^{-1}$, and a maximum value of $333 \times 10^{-3} \mathrm{~kg} \cdot \mathrm{km}^{-1} \cdot \mathrm{h}^{-1}$ for CO and $37 \times 10^{-3} \mathrm{~kg} \cdot \mathrm{km}^{-1} \cdot \mathrm{h}^{-1}$ for PM10 at 18:00, for a total of 617 vehicles. $h^{-1}$. 
The two sides of the LP Avenue have different characteristics: while the two Northern lanes are one of the entrances to the city for traffic coming from a national road (N109) and neighbouring localities and towns, the other two represent an important connection between the city centre and the services and commerce in the Avenue. In order to construct the traffic scenarios (section 3.5) an analysis of traffic flux in each side of the Avenue was made. The results shown in Figure 4 represent the total hourly mean traffic in weekdays in the Northern and Southern lanes. As can be seen, a higher flux occurs in the South part of the Avenue. In average, the flux in the Southern lanes is 37\% higher than the one in the opposite sense. The highest differences are found for the period from 7:00 to 19:00.

\subsection{Baseline simulations and scenarios}

The simulations were performed for the baseline situation, considering current conditions in terms of typical meteorology and traffic flux for this area. Two distinct traffic alternatives were evaluated under different meteorological conditions. In the first the traffic was maintained only in the two lanes located in the North side of the Avenue, while in the second the opposite was considered. It was assumed that these planning solutions do not lead to a modification of the current traffic fluxes. The reason for this assumption is that there are no apparent reasons justifying the increase or decrease of the flux in a particular sense because of the restriction imposed to the opposite sense, in particular having in mind the distinct roles of each side as previously referred in section 3.4.

Table 1 shows the 6 scenarios considered based on the combination of different traffic emissions and meteorological conditions.

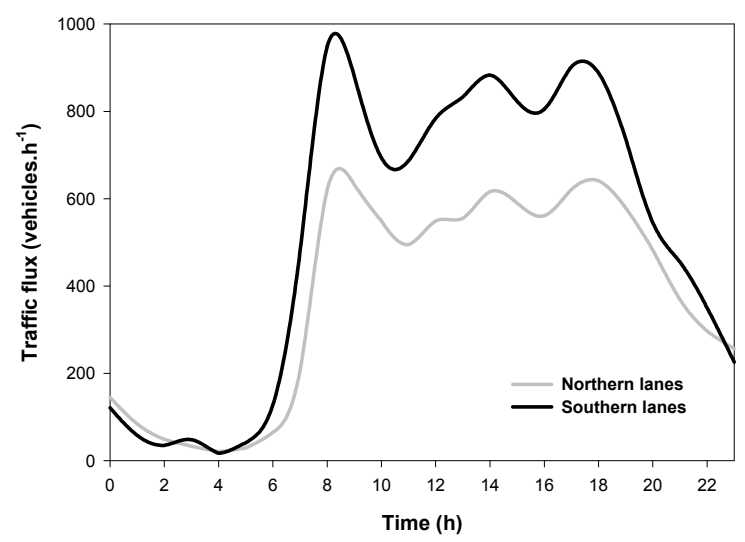

Figure 4: Time evolution of the hourly number of vehicles in each sense of the LP Avenue. 
Table 1: $\quad$ Scenarios development. Road traffic alternative North indicates that only the traffic in the Northern lanes is allowed. The opposite for the South is the alternative.

\begin{tabular}{|c|c|c|c|c|c|c|c|c|}
\hline \multirow{3}{*}{$\begin{array}{c}\text { Scenario } \\
\#\end{array}$} & \multirow{3}{*}{$\begin{array}{l}\text { Road } \\
\text { traffic } \\
\text { alternative }\end{array}$} & \multirow{3}{*}{$\begin{array}{l}\text { Time of } \\
\text { the day }\end{array}$} & \multicolumn{2}{|c|}{$\begin{array}{l}\text { Meteorological } \\
\text { conditions }\end{array}$} & \multicolumn{4}{|c|}{$\begin{array}{l}\text { Road traffic emissions in } \\
\text { the LP Avenue } \\
\left(\times 10^{-3} \mathrm{~kg} \cdot \mathrm{km}^{-1} \cdot \mathrm{h}^{-1}\right)\end{array}$} \\
\hline & & & Mean & $\begin{array}{l}\text { Mean } \\
\text { wind }\end{array}$ & $\begin{array}{l}\text { North } \\
\text { sense }\end{array}$ & $\begin{array}{l}\text { North } \\
\text { sense }\end{array}$ & $\begin{array}{l}\text { South } \\
\text { sense }\end{array}$ & $\begin{array}{l}\text { South } \\
\text { sense }\end{array}$ \\
\hline & & & direction & $\begin{array}{l}\text { velocity } \\
\left(\mathrm{m} \cdot \mathrm{s}^{-1}\right)\end{array}$ & $\mathrm{CO}$ & PM10 & $\mathrm{CO}$ & PM10 \\
\hline 1 & North & Morning & $\begin{array}{c}\text { NW } \\
\left(315^{\circ}\right)\end{array}$ & 2.64 & 127 & 14 & 186 & 20 \\
\hline 2 & South & Morning & $\begin{array}{c}\text { NW } \\
\left(315^{\circ}\right)\end{array}$ & 2.64 & 127 & 14 & 186 & 20 \\
\hline 3 & North & Morning & $\begin{array}{c}\mathrm{SE} \\
\left(225^{\circ}\right)\end{array}$ & 2.64 & 127 & 14 & 186 & 20 \\
\hline 4 & South & Morning & $\begin{array}{c}\mathrm{SE} \\
\left(225^{\circ}\right)\end{array}$ & 2.64 & 127 & 14 & 186 & 20 \\
\hline 5 & North & Afternoon & $\begin{array}{c}\text { NW } \\
\left(315^{\circ}\right)\end{array}$ & 4.59 & 275 & 30 & 369 & 41 \\
\hline 6 & South & Afternoon & $\begin{array}{c}\text { NW } \\
\left(315^{\circ}\right)\end{array}$ & 4.59 & 275 & 30 & 369 & 41 \\
\hline
\end{tabular}

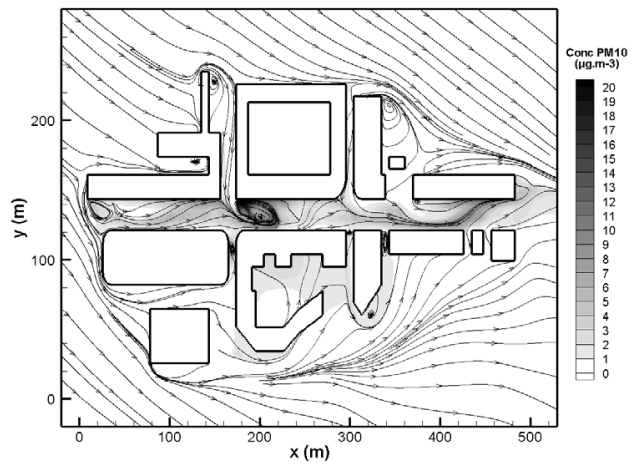

Figure 5: $\quad 1.5 \mathrm{~m}$ high horizontal PM10 concentration field and streamlines for the baseline situation, with typical traffic fluxes and meteorological conditions in the afternoon. 


\section{Modelling results analysis}

Figure 5 shows the horizontal PM10 concentration fields, for a height of $1.5 \mathrm{~m}$ (the typical value for exposure studies), corresponding to the baseline situation (in afternoon conditions). The figure shows a hot-spot at the centre of the Avenue due to a vortex induced by the incoming flow from the transversal street. The maximum PM10 concentration in the domain is $22 \mu \mathrm{g} \cdot \mathrm{m}^{-3}$, while for CO is $200 \mu \mathrm{g} . \mathrm{m}^{-3}$. If the mean concentration is calculated for a line crossing the domain along the Avenue's sidewalks, distanced $1 \mathrm{~m}$ from the walls and at $1.5 \mathrm{~m} \mathrm{high}$, the following average results are obtained: $47 \mu \mathrm{g} . \mathrm{m}^{-3}$ of $\mathrm{CO}$ and $5 \mu \mathrm{g} \cdot \mathrm{m}^{-3}$ of PM10 in the North sidewalk, and $31 \mu \mathrm{g} . \mathrm{m}^{-3}$ of $\mathrm{CO}$ and $3 \mu \mathrm{g} \cdot \mathrm{m}^{-3}$ of PM10 in the South sidewalk, which shows a larger accumulation of pollutants in the North side of the street-canyon for NW wind conditions.

Figure 6 shows the horizontal PM10 concentration fields, for a height of 1.5 $\mathrm{m}$, corresponding to scenarios 5 (road traffic only in the North part of the domain), and 6 (road traffic only in the South part of the domain).

(a)

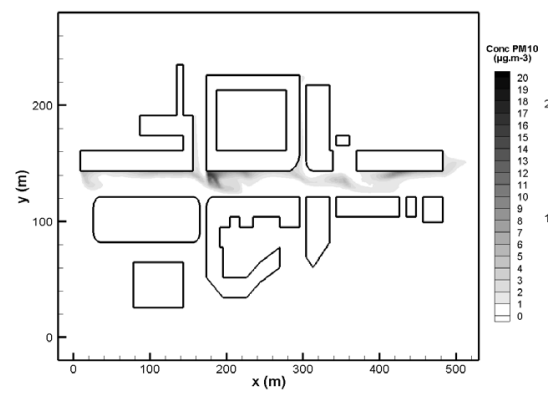

(b)

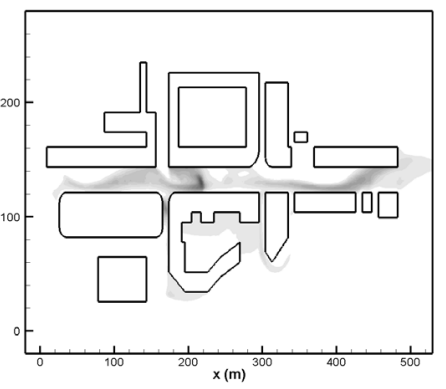

Figure 6: Comparison of $1.5 \mathrm{~m}$ high horizontal PM10 concentration fields during the afternoon for (a) scenario 5 (road traffic only in the North part of the domain), and (b) scenario 6 (road traffic only in the South part of the domain).

For the situation shown in Figure 6, keeping the traffic only in the North side (scenario 5) leads to a decrease of $27 \%$ of the maximum PM10 concentration attained, while the other solution (scenario 6) offers a decrease of $36 \%$. Table 2 presents a comparison between the maximum concentrations attained in the study domain for the present conditions and for each scenario considered. In order to allow a better understanding on the effect over the maximum concentrations, the values for CO and PM10 were normalized by the maximum concentration for the respective pollutant given by the baseline simulation. Results are given by the non-dimensional parameter $\mathrm{C}$, given by equation (1):

$$
C=\frac{[X]_{\text {scenario\# }}}{[X]_{\text {baseline }}}
$$


Table 2: $\quad$ Maximum CO and PM10 concentrations in the study domain (at $1.5 \mathrm{~m}$ high) for the different baseline and scenarios.

\begin{tabular}{ccccc}
\hline Simulation & \multicolumn{2}{c}{$\begin{array}{c}\text { Maximum CO } \\
\text { concentration }\end{array}$} & \multicolumn{2}{c}{$\begin{array}{c}\text { Maximum PM10 } \\
\text { concentration }\end{array}$} \\
& $\left(\mu \mathrm{g} . \mathrm{m}^{-3}\right)$ & $\mathrm{C}_{\mathrm{CO}}(-)$ & $\left(\mu \mathrm{g} . \mathrm{m}^{-3}\right)$ & $\mathrm{C}_{\mathrm{PM} 10}(-)$ \\
\hline Morning, NW wind: & 195 & 1.00 & 22 & 1.00 \\
Baseline & 151 & 0.77 & 18 & 0.82 \\
Scenario 1 & 108 & 0.55 & 12 & 0.55 \\
Scenario 2 & 153 & 1.00 & 17 & 1.00 \\
Morning, SE wind: & 66 & 0.43 & 7 & 0.41 \\
Baseline & 129 & 0.84 & 14 & 0.82 \\
Scenario 3 & 200 & 1.00 & 22 & 1.00 \\
Scenario 4 & 149 & 0.75 & 16 & 0.73 \\
Afternoon, NW wind: & 126 & 0.63 & 14 & 0.64 \\
Baseline & & & & \\
Scenario 5 & Scenario 6 & &
\end{tabular}

where $[\mathrm{X}]$ is the maximum air pollutant concentration at $1.5 \mathrm{~m}$ high. It can be inferred from the analysis of the table that while for NW winds the best solution for the reduction of the maximum concentrations is to restrict the North area to traffic, the opposite occurs for SE conditions. The maximum reduction is found for scenario 3, according to which decreases of 57\% and 59\%, respectively, on the maximum $\mathrm{CO}$ and PM10 concentrations are obtained.

However, although the restriction to traffic in the North side (scenario 6) results on a decrease on maximum concentrations, a general decrease of air quality is observed in the study area as can be seen in Figure 6. This fact is explained by the characteristics of the wind vortex formed in the street-canyon for typical meteorological conditions.

In this sense, the comparative analysis of the effect of traffic scenarios on air pollutants concentrations was also made by calculating the mean concentration in a line distanced $1 \mathrm{~m}$ from each sidewalk of the LP Avenue and at $1.5 \mathrm{~m} \mathrm{high}$. Concentrations were also normalized by the mean concentration in each sidewalk derived from the baseline simulation, according to eqn. (1). In this case, [X] is the mean concentration value in the sidewalk horizontal line.

The results obtained for the 6 scenarios are represented in Figure 7 for PM10 concentration. The grey bar represents the normalized PM10 concentration in the North sidewalk, while the black bar corresponds to the South sidewalk. Comparisons should be made between scenarios 1 and 2 (morning conditions, with NW wind), 3 and 4 (morning conditions, with SE wind), and 5 and 6 (afternoon conditions, with NW wind).

The figure shows that, for the same meteorological conditions, closing the south part of the Avenue and adjacent streets to traffic would return better results in terms of air quality improvement. In fact, the comparison between scenarios 1 and 2, and 5 and 6, reveals an important reduction on PM10 concentration in the 


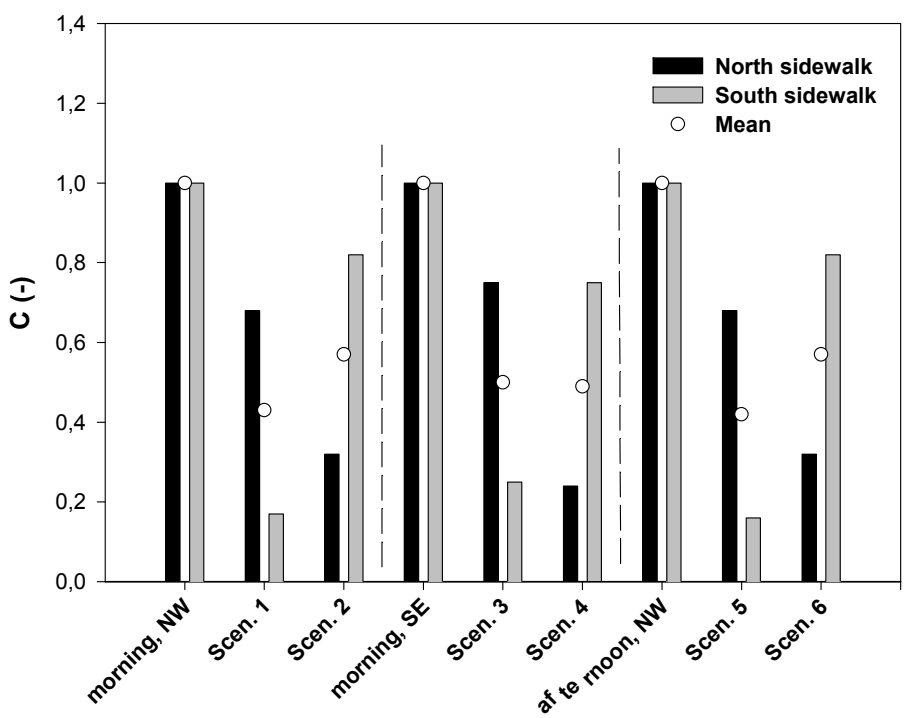

Figure 7: Comparative analysis of normalized mean PM10 concentrations in both sidewalks of LP Avenue, and mean values for each scenario.

South sidewalk when only the traffic in the North part of the domain is considered. This effect is not significant for the morning situation with SE winds, for which scenarios 3 and 4 have similar mean values. Scenario 5 is the one for which the air quality improvement is larger: the decrease on PM10 equals $32 \%$ in the North sidewalk and $84 \%$ in the South sidewalk. The increase of the wind speed from the morning to the afternoon, with NW wind direction, does not have a considerable effect on results, as can be concluded by comparing scenarios 1 with 5 , and 2 with 6 . Same conclusions were taken for CO.

The comparative analysis cannot neglect the fact that, for a typical day, the road traffic flux in the Southern part of the LP Avenue is in average 37\% higher than in the other sense. In order to eliminate this factor from the analysis the mean concentrations in both sidewalks were divided by the mean traffic flux for that particular traffic sense, according to the following equation:

$$
C^{*}=\frac{[X]}{F}
$$

where $\mathrm{C}^{*}$ has units of $\mu \mathrm{g} \cdot \mathrm{m}^{-3} \cdot$ vehic $^{-1} \cdot \mathrm{h}^{-1}$, and $\mathrm{F}$ is the hourly mean traffic flux. Results are shown in Figure 8.

According to the data shown in the previous figure, for identical traffic fluxes the alternative of restricting the traffic in the Northern part of the domain is always the most advantageous in terms of the air pollution levels in the sidewalks. The explanation for this is that, in fact, from the point of view of the wind flow characteristics inside the street-canyon, and the resulting ventilation capacity, an enhanced dispersion of the air pollutants emitted is obtained if the vehicles circulation is restricted to the south area (for typical NW and SE winds). 


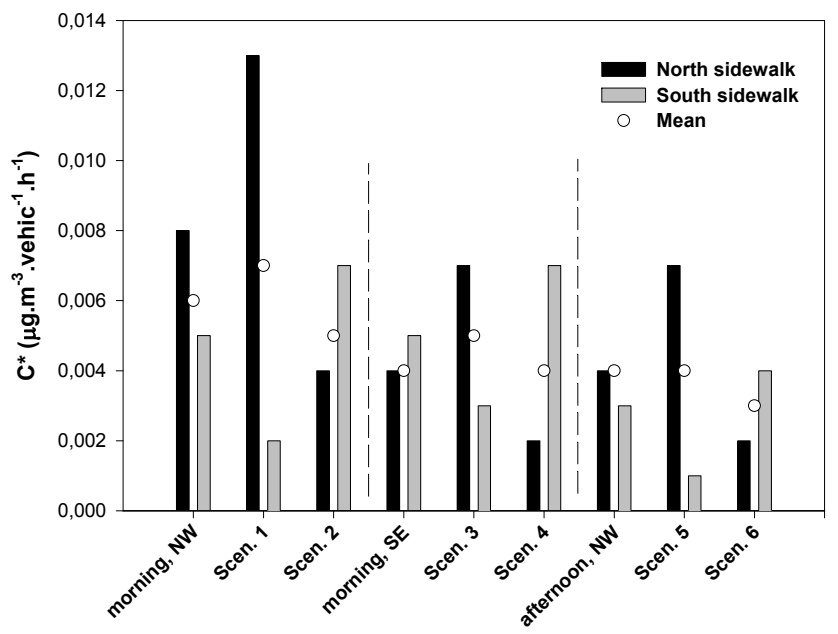

Figure 8: Comparative analysis of PM10 concentrations per traffic flux in both sidewalks of LP Avenue and mean values for baseline and scenarios.

\section{Conclusions}

The CFD simulations have shown that, for typical meteorological conditions, a complex wind flow is induced by the positioning of buildings and trees in the analysed street-canyon. Distinct behaviours of the air pollutants inside the study domain were found for the different scenarios due to the prevailing wind direction and the location of the emission sources.

If the same traffic flux is assumed in both sides of the main Avenue, the behaviour of the wind flow in the study area leads to lower concentrations in the sidewalks if the circulation of vehicles in the North part of the study area is restricted. However, taking into account the currently distinct fluxes of the two senses (higher in the Southern lanes), and assuming that no changes occurs in fluxes if one of the senses is closed, the traffic restriction of the two traffic lanes located in the South side of the Avenue canyon leads to a $31 \%$ average reduction on CO and PM10 concentrations in the North sidewalk and $81 \%$ in the South sidewalk when comparing to present conditions. The other alternative leads to a reduction of, respectively, $70 \%$ and $20 \%$. The first scenario leads also to maximum decreases of $57 \%$ and 59\%, respectively, on the CO and PM10 peak concentrations. From the point of view of air quality improvement the elimination of the Southern lanes is therefore the most advantageous solution.

These conclusions stress the importance of integrating the knowledge on road traffic management, urban planning, and air quality as driving forces towards urban sustainability. With this goal, the application of CFD models in the detailed simulation of air pollutants dispersion in cities under varying conditions is currently an approach with increasing potential. 


\section{Acknowledgements}

The authors would like to acknowledge the financial support of the $3^{\text {rd }}$ European Framework Program and the Portuguese Ministry of Science, Technology and Higher Education for the Post-Doc grant of J.H. Amorim (SFRH/BPD/48121/2008) and for the $\mathrm{PhD}$ grant of $\mathrm{R}$. Tavares (SFRH/BD/22741/2005). The authors are also grateful to the European research Project BRIDGE (211345/EC).

\section{References}

[1] EEA, Europe's environment - The fourth assessment, Office for Official Publications of the European Communities, European Environmental Agency: Luxembourg, 452 p., 2007.

[2] WHO, Health Aspects of Air Pollution Results from the WHO Project "Systematic Review of Health Aspects of Air Pollution in Europe", World Health Organization: Geneva, 2004.

[3] Prüss-Üstün, A. \& Corvalán, C., Preventing disease through healthy environments. Towards an estimate of the environmental burden of disease. World Health Organization: Geneva, 2006.

[4] Borrego, C., Lopes, M., Valente, V., Neuparth, N., Martins, P., Amorim, J.H., Costa, A.M., Silva, J., Martins, H., Tavares, R., Nunes, T., Miranda, A.I., Cascão, P. \& Ribeiro, I., The importance of urban planning on air quality and human health (Chapter 2). Urban Planning in the $21^{\text {st }}$ Century, eds. D.S. Graber \& K.A. Birmingham, Nova Science Publishers Inc., 2009.

[5] Oke, T.R., Street design and urban canopy layer climate. Energy and Buildings, 11(1-3), pp. 103-113, 1988.

[6] Borrego, C., Tchepel, O., Costa, A.M., Amorim, J.H. \& Miranda, A.I., Emission and dispersion modelling of Lisbon air quality at local scale. Atmospheric Environment, 37, pp. 5197-5205, 2003.

[7] Martins, A., Cerqueira, M., Ferreira, F., Borrego, C. \& Amorim J.H., Lisbon air quality - evaluating traffic hot-spots. International Journal of Environment and Pollution, 39(3/4), pp. 306-320, 2009.

[8] Amorim, J.H., Rodrigues, V., Borrego, C. \& Costa A.M., A CFD analysis of the vegetative canopy effect on urban air pollutants dispersion. Proc. of the International Conference on Local Air Quality and its Interaction with Vegetation. pp. 104-108, 2010.

[9] Fluent Inc., FLUENT 6.1 User's manual, Fluent Inc.: Lebanon, 2003. 